\title{
Isolation and Identification of Listeria monocytogenes from Fish Intestines and Phylogenetic Analysis
}

\author{
N. Madharsha ${ }^{1}$, Veeramanikandan Veeramani ${ }^{1 *}$, \\ Jaianand Kannaiyan ${ }^{2}$ and Balaji Paulraj ${ }^{2}$ \\ ${ }^{1}$ Department of Microbiology, ${ }^{2}$ Department of Biotechnology, MGR College, Dr. MGR Nagar, \\ Hosur, Tamil Nadu, India \\ *Corresponding author
}

\begin{abstract}
A B S T R A C T
\end{abstract}

\begin{tabular}{|l|}
\hline K e y w o r d s \\
$\begin{array}{l}\text { Listeriosis, Listeria } \\
\text { monocytogenes, Labeo catla } \\
\text { (Catla fish), Dasyatis } \\
\text { pastinaca (Stingrays fish), } \\
\text { Phylogenetic analysis }\end{array}$ \\
\hline Article Info \\
\hline $\begin{array}{l}\text { Accepted: } \\
10 \text { October } 2018 \\
\text { Available Online: } \\
10 \text { November } 2018\end{array}$ \\
\hline
\end{tabular}

\section{Introduction}

Listeriosis is one of the developing zoonotic foodborne illnesses commonly acquired through consumption of contaminated foods particularly foods of animal origin. Listeria species could spread the food supply chain over horizontal transmission and spoil the products like fish, chicken and meat products at any period of production, processing, distribution, marketing, handling and preparation of foods (Alsheikh et al., 2012). Recently people are concerned to preserve the fish and meat products under cold temperature, in order to extend the shelf life or to make food at their suitable time. But, refrigeration condition and storage which inhibit the other contaminate organisms rather preserve and promote the survival of Listeria species in the fish, meat or meat products, thus endangering the health of consumers (Walker et al., 1990; Farber and Peterin., 1991).

It also increases the growth environments conducive for psychotropic pathogens such as Listeria, letting them grow to unsafe levels in meat or other products (Francis and $\mathrm{O}^{\mathrm{ee} B e i r n e,}$ 1998). These lead to the presence of Listeria species in different fish, meat and meat products in diverse geographical regions and promote causing in foodborne outbreaks (Borucki, 2003). 
On the other side, the development of a rapid, precise and perceptive typing method is needed to observe Listeriosis outbreaks.

Of the various typing techniques established to differentiate Listeria at subspecies level, serotyping was the utmost usually and widely used (Seeliger et al., 1979). Though, the limited value of serotyping in Listeria typing was stated in several studies, stemming from the fact that only a minority of serovars were detected from field isolates (Schuchat et al., 1991). Similarly, various reports can be shown in the presence of L. monocytogenes in fish and fish products (Byun et al., 2001 and Jrgensen et al., 1998). Keeping in view the above facts in the present study an attempt was made to investigate the prevalence of Listeria spp. and its classification of phylogenetic relationship characterization from two different variety of fish collected from markets/ retail shops as well as roadside vendors located in and around West Chennai.

\section{Materials and Methods}

\section{Sample collection}

In the present study totally 40 fish samples were analyzed. The nature of the samples collected included the nature of retail outlets such as selling of fish of single species and/or multiple species, processed products and unbranded categories as relevant to the fish samples. The Study period was December 2017 to February 2018. In brief, a total of 100 samples comprising Catla fish (Labeo catla) $(\mathrm{n}=20)$ and Stingrays fish (Dasyatis pastinaca) $(n=20)$, were randomly collected from the selected retail shop in Virugambakkam location in West Chennai city. The fish were placed into separate plastic bags in the icebox at $4^{\circ} \mathrm{C}$ and immediately transferred to the Microbiology Laboratory at MGR College, Hosur. Each fresh fish was opened with a sterile scalpel and about $1 \mathrm{~g}$ of intestine content was taken using a sterile swab for Listeria isolation.

\section{Standard strains}

The standard strain of Listeria monoctogenes (MTCC 1143), used in the study were procured from the microbial Type culture collection and gene bank, Institute of microbial technology, Chandigarh (IMTC), India.

\section{Isolation and identification of Listeria sp.}

ISO 11290 method was employed to isolate the organisms, whereby pre-enrichment of 25 g sample was done in $225 \mathrm{ml}$ half strength Fraser broth containing selective supplements (Hi-Media) for $24 \mathrm{~h}$ at $30{ }^{\circ} \mathrm{C}$, which was followed by second enrichment of $0.1 \mathrm{ml}$ of pre-enriched Fraser broth content in $10 \mathrm{ml}$ full strength Fraser containing selective supplements (Hi-Media) for $48 \mathrm{~h}$ at $37{ }^{\circ} \mathrm{C}$ incubation temperature. After the enrichment procedure, the inoculum was plated on PALCAM agar (Hi-Media) and incubated for $48 \mathrm{~h}$ at $37^{\circ} \mathrm{C}$.

The gray-green colonies surrounded by diffuse black zone on Polymyxin Acriflavin Lithium Chloride Ceftazidime Aesculin Mannitol (PALCAM) agar was picked up and further purified on Tryptone Soya Yeast Extract Agar (TSYEA). Subsequently pinpoint colonies of TSYEA were subjected to identification procedures which included Gram' Staining followed by a microscopic examination, catalase test, and oxidase test. The characteristic Gram-positive, short rod-shaped organisms which were catalase positive and oxidase negative, were subcultured in TSYEA agar at $25{ }^{\circ} \mathrm{C}$ for 12 - $18 \mathrm{~h}$. subsequently, culture showing typically tumbling motility were considered as "presumptive" Listeria isolates, which were in turn subjected to detailed biochemical tests viz.; methyl red, 
Voges-Proskauer test, nitrate, and sugar fermentation tests with xylose, mannitol, glucose, galactose, maltose, lactose, fructose.

\section{In-vitro pathogenicity tests}

The isolates were tested for the type and the degree of hemolysis on $5 \%$ sheep blood agar and the Christie Atkins Munch Peterson (CAMP test) was conducted using Listeria isolates, $S$. aureus, standard strains to observe for hemolysis between Listeria strain and the $S$. aureus owing to the synergistic effect of their hemolysins in case of a CAMP-positive reaction. In detail for Hemolysis test, inoculated heavily from TSYEA onto sheep blood agar by stabbing the plates by passing through the agar layer and incubated at 48 hours at $35^{\circ} \mathrm{C}$. The positive result for Listeria monocytogenes was produce slightly cleared zone around stab. On the other hand, for CAMP test streaked the hemolytic Staphylococcus aureus and were cultured in parallel and diametrically opposite to each other on a sheep blood agar plate, streak several test culture parallels to one another, but at right angles to and in between Staphylococcus aureus streaks. After incubation at $35^{\circ} \mathrm{C}$ for $24-48$ hours the plates were examined for hemolysis. Listeria monocytogenes reaction is often optimal at 24 hours rather than 48 hours.

\section{S rDNA sequence determination}

A colony PCR method was used for amplification of 16S rDNA. A single colony of the bacterial isolate was suspended into the $100 \mu \mathrm{l}$ sterile distilled water. Boiled for 25 minutes at $94^{\circ} \mathrm{C}$ and centrifuged at maximum speed in a microcentrifuge for 15 minutes to pellet cell debris. $5 \mu \mathrm{l}$ of the supernatant was used as DNA templates for PCR. Bacterial $16 \mathrm{~S}$ rDNA was amplified from the extracted genomic DNA by using the universal bacterial $16 \mathrm{~S}$ rDNA primers $27 \mathrm{~F}$ forward primer (5'
AGAGTTTGATCMTGGCTCAG 3') and 1492R reverse primer (5'

TACGGYTACCTTGTTACGACTT 3'). PCR was performed with a $25 \mu$ l reaction mixture containing $5 \mu \mathrm{l}$ of DNA extract as the template, $1.5 \mu \mathrm{L}$ of Forward Primer and Reverse Primer, $5 \mu \mathrm{L}$ of deionized water, and $12 \mu \mathrm{L}$ of Taq Master Mix. Removed unincorporated PCR primers and dNTPs from PCR products by using Montage PCR Clean-up kit (Millipore).

\section{PCR amplification}

The PCR product was sequenced using the primers. Sequencing reactions were performed using an ABI PRISM BigDyeTM Terminator Cycle Sequencing Kits with AmpliTaq DNA polymerase (FS enzyme) (Applied Biosystems) and consists of an initial $94^{\circ} \mathrm{C}$ denaturation for $3 \mathrm{~min}$ followed by 30 cycles of $94^{\circ} \mathrm{C}$ for $30 \mathrm{sec}, 60^{\circ} \mathrm{C}$ for $30 \mathrm{sec}, 72^{\circ} \mathrm{C}$ for 1 min, followed by a final extension at $72^{\circ} \mathrm{C}$ for 10 min. Single-pass sequencing was performed on each template using below $16 \mathrm{~s}$ rRNA universal primers. The fluorescentlabeled fragments were purified from the unincorporated terminators with an ethanol precipitation protocol. The samples were resuspended in distilled water and subjected to electrophoresis in an ABI 3730xl sequencer (Applied Biosystems).

Nucleotide sequencing, alignment, and phylogeny

The 16s rRNA sequence was blast using NCBI blast similarity search tool. The phylogeny analysis of query sequence with the closely related sequence of blast results was performed followed by multiple sequence alignment. Based on the scoring index, the program MUSCLE 3.7 was used for multiple alignments of sequences (Edgar et al., 2004). The resulting aligned sequences were cured using the program Gblocks 0.91b. This Gblocks eliminates poorly aligned positions 
and divergent regions (Talavera et al., 2007). Finally, the program PhyML 3.0 aLRT was used for phylogeny analysis and HKY85 as substitution model. PhyML was shown to be at least as accurate as other existing phylogeny programs using simulated data while being one order of magnitude faster. The program Tree Dyn 198.3 was used for tree rendering (Edwards et al., 1989).

\section{Results and Discussion}

The study was undertaken to the occurrence of Listeria species in fish samples form Labeo catla and Dasyatis pastinaca $(\mathrm{n}=40)$ which were collected from different retail outlets located in and around from West Chennai (Figure 1). Samples were processed following the reference method ISO 11290:1996 with respect to isolation, identification and biochemical confirmation of Listeria species. Samples were broadly categorized based on the species slaughtered and type of processing at the retail outlets (Table 1).

\section{The occurrence of Listeria species in fish}

Colonies showing typical cultural and morphological characteristics such as greygreen shiny colonies having diffused black shadow were considered as characteristic of Listeria species on the selective PALCAM agar plates. Further, these colonies were streaked onto TSYEA slants and used for the biochemical analysis. The results of cultural (Figure 2 and 3) and biochemical reactions are shown in Table 2 and 3. Putative isolates showing typical biochemical reactions were confirmed. Of the total 20 Catla fish and 20 Stingrays fish samples analyzed for the presence of Listeria species using ISO11290:1996 method of isolation and identification and 20 samples showed the presence of Listeria monocytogenes and with an overall occurrence of $50 \%$ of Listeria monocytogenes in fish samples.

\section{S rDNA sequence determination}

Based on the morphological, physiological, biochemical characteristics (Table 2 and 3) showed that the ATCC 51779 strain is matched to the members of genus Listeria monocytogenes; accession number MH701890. The partially amplified for two different unknown samples correspondingly $978 \mathrm{bp}$ and $1045 \mathrm{bp}$ fragment of $16 \mathrm{~S}$ rDNA sequence was submitted to NCBI database search using BLASTN to confirm the species of the bacterium. The highest sequence similarity revealed that sample from Labeo catla is closely related to Listeria monocytogenes; accession number MH701866, and similarly sample from Dasyatis pastinaca is closely related to Listeria monocytogenes; accession number MH701867.

Nucleotide sequencing, alignment, and phylogeny

The multiple sequence alignment and the phylogenetic relationship confirmed the highest sequence similarity with the ATCC 51779 strain Listeria monocytogenes (KF956740.1) with a distribution of the top 200 blast hits.

In contrast, the unknown samples were confirmed the highest sequence similarity with Listeria monocytogenes (CP011398.2) and (CP028411.1) with a distribution of the top 200 blast hits on 100 subject sequences for Labeo catla and Dasyatis pastinaca respectively.

The calculation and classification of a phylogenetic relationship was determined and PhyML was shown to be at least as accurate as other existing phylogeny programs using simulated data while being one order of magnitude faster (Figure 4). The program Tree Dyn 198.3 was used for tree rendering. 
Fig.1 Appearance of raw material for the occurrence of Listeria species in fish samples (A) Labeo catla, (B) Dasyatis pastinaca

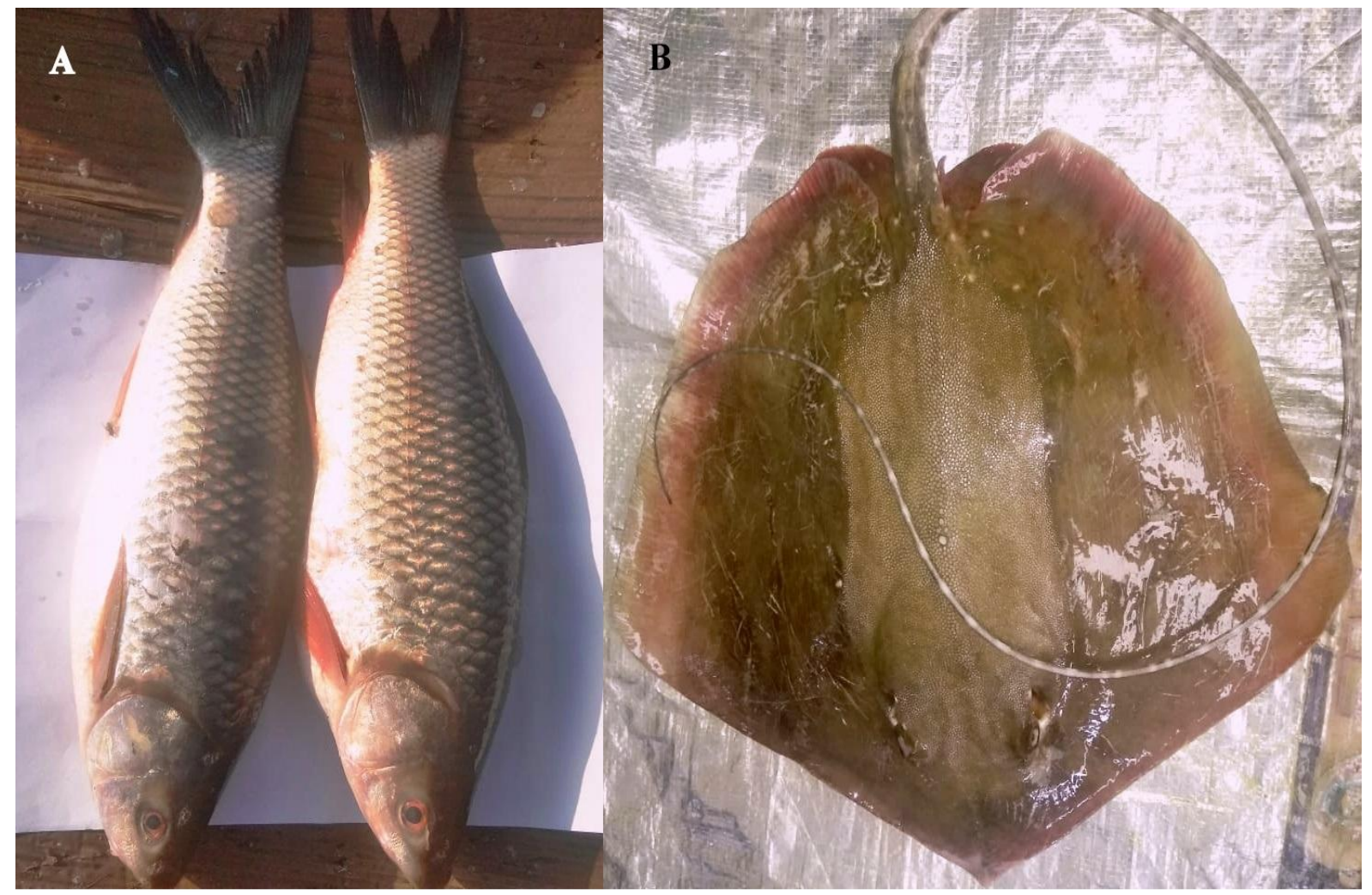

Fig.2 Identification of Listeria species in Labeo catla. A representative result of (A) The microbial strain grown on Oxford and PALCAM agar plate, (B) TSYEA plate, (C) Blood agar Christie Atkins Munch Peterson test, (D) Isolates from Labeo catla grown on blood agar
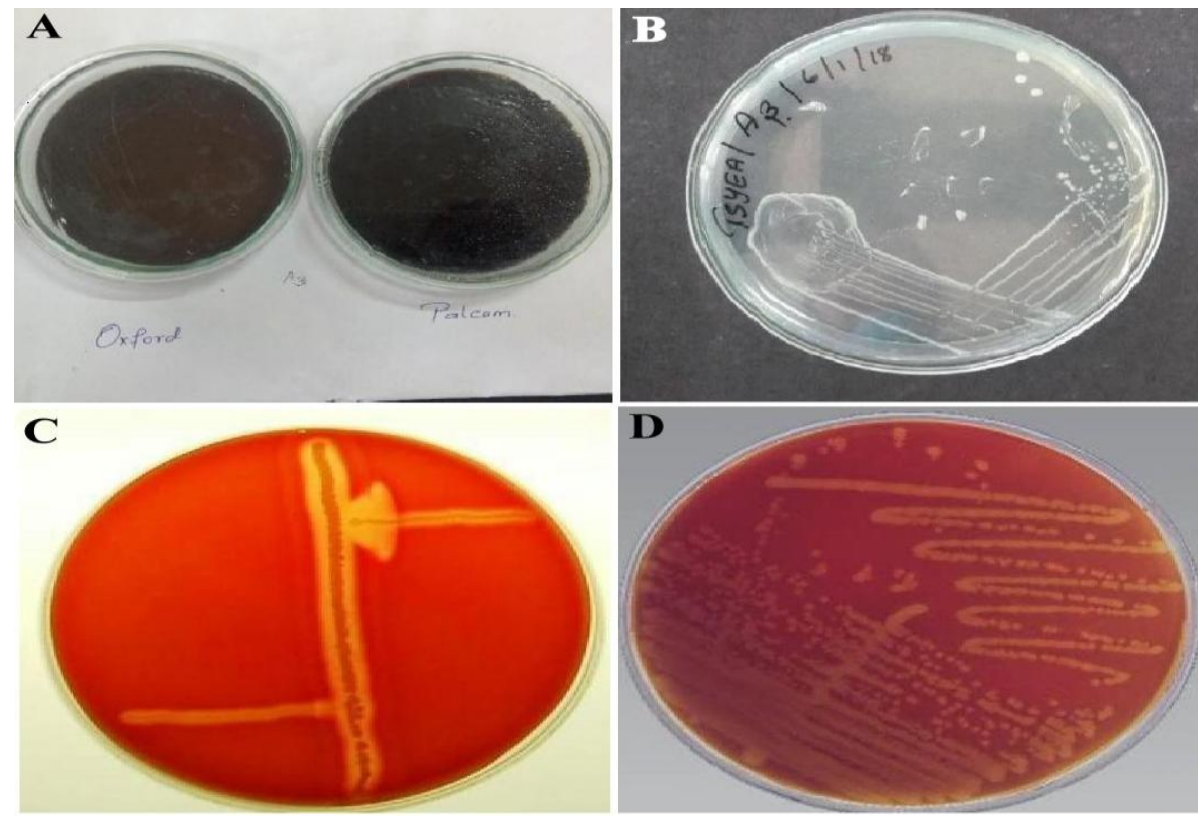
Fig.3 Identification of Listeria species in Dasyatis pastinaca. A representative result of (A) The microbial strain grown on Oxford and PALCAM agar plate, (B) TSYEA plate, (C) Blood agar Christie Atkins Munch Peterson test, (D) Isolates from Dasyatis pastinaca grown on blood agar
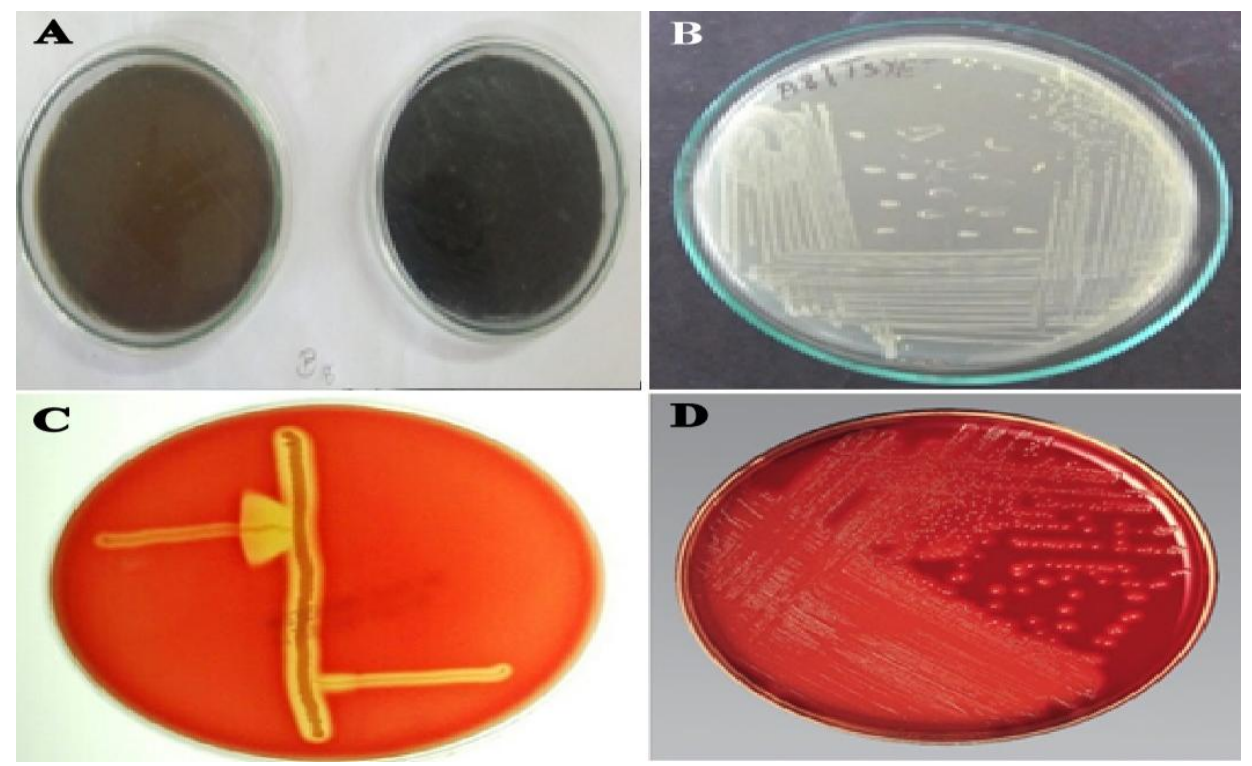

Fig.4 The phylogenetic relationship based on 16S rDNA sequence comparison showing the position of Listeria monocytogenes ATCC 51779 strain (KF956740.1), Labeo catla

(CP011398.2), and Dasyatis pastinaca (CP028411.1). The GeneBank accession numbers for the $16 \mathrm{~S}$ rDNA sequences are given after the strain in parenthesis. While scale bar corresponds to nucleotide sequence difference

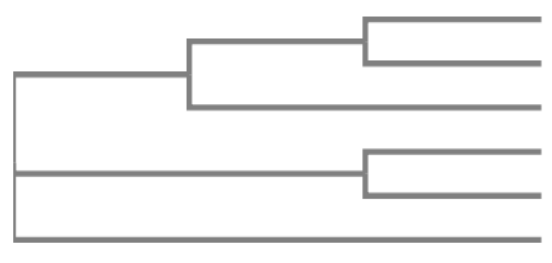

_Listeria_monocytogenes

KF956740.1_Listeria_monocytogene CP029372.1_Listeria_monocytogene CP020828.1_Listeria_monocytogene CP028412.1_Listeria_monocytogene CP007171.1_Listeria_monocytogene

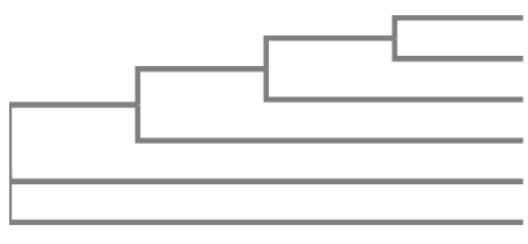

CP011398.2_Listeria_monocytogene CP028412.1_Listeria_monocytogene Listeria_monocytogenes MH532494.1_Listeria_monocytogene MF970454.1_Listeria_sp. MF970451.1_Listeria_sp.

CP028411.1_Listeria_monocytogene CP029372.1_Listeria_monocytogene CP028413.1_Listeria_monocytogene CP028412.1_Listeria_monocytogene _Listeria_monocytogenes MF784284.1_Listeria_monocytogene 
Table.1 Collection of samples for isolation of Listeria species in fish

\begin{tabular}{|c|c|c|c|}
\hline Fish Type & Nature & Location & Collection (Nos) \\
\hline \multirow{2}{*}{$\begin{array}{l}\text { Catla } \\
\text { (Labeo } \\
\text { catla) }\end{array}$} & $\begin{array}{l}\text { Outlet selling only Fish } \\
\text { (single species) }\end{array}$ & Chennai West & 10 \\
\hline & $\begin{array}{l}\text { Outlet selling Fish } \\
\text { (multiple species) }\end{array}$ & Chennai West & 10 \\
\hline \multirow{2}{*}{$\begin{array}{l}\text { Stingrays } \\
\text { (Dasyatis } \\
\text { pastinaca) }\end{array}$} & $\begin{array}{l}\text { Outlet selling only Fish } \\
\text { (single species) }\end{array}$ & Chennai West & 10 \\
\hline & $\begin{array}{l}\text { Outlet selling Fish } \\
\text { (multiple species) }\end{array}$ & Chennai West & 10 \\
\hline
\end{tabular}

Table.2 Cultural and biochemical characteristics of Listeria species isolated from Labeo catla

\begin{tabular}{|c|c|c|c|c|c|c|c|c|c|c|c|c|c|c|c|c|c|c|c|c|c|}
\hline Labeo catla Isolates & ATCC & A1 & A2 & A3 & A4 & A5 & A6 & A7 & A8 & A9 & A 10 & A 11 & A 12 & A 13 & A 14 & A 15 & A 16 & A 17 & A 18 & A 19 & A 20 \\
\hline Gram's staining & $\begin{array}{l}(\mathrm{P}), \\
\text { rods }\end{array}$ & $\begin{array}{l}(\mathrm{P}), \\
\text { rods }\end{array}$ & $\begin{array}{c}(\mathrm{P}) \\
\text { coccus }\end{array}$ & $\begin{array}{l}(\mathrm{P}), \\
\text { rods }\end{array}$ & $\begin{array}{l}(\mathrm{N}), \\
\text { rods }\end{array}$ & $\begin{array}{l}(\mathrm{P}), \\
\text { rods }\end{array}$ & $\begin{array}{c}(\mathrm{P}), \\
\text { coccus }\end{array}$ & $\begin{array}{l}(\mathrm{P}), \\
\text { rods }\end{array}$ & $\begin{array}{c}(\mathrm{P}), \\
\text { coccus }\end{array}$ & $\begin{array}{l}(\mathrm{P}) \\
\text { rods }\end{array}$ & $\begin{array}{l}(\mathrm{N}) \\
\text { rods }\end{array}$ & $\begin{array}{l}(\mathrm{P}) \\
\text { rods }\end{array}$ & $\begin{array}{l}(\mathrm{P}), \\
\text { rods }\end{array}$ & $\begin{array}{l}(\mathrm{P}), \\
\text { rods }\end{array}$ & $\begin{array}{c}(\mathrm{P}), \\
\text { coccus }\end{array}$ & $\begin{array}{l}(\mathrm{P}), \\
\text { rods }\end{array}$ & $\begin{array}{l}(\mathrm{N}) \\
\text { rods }\end{array}$ & $\begin{array}{l}(\mathrm{P}), \\
\text { rods }\end{array}$ & $\begin{array}{c}(\mathrm{P}), \\
\text { coccus }\end{array}$ & $\begin{array}{l}(\mathrm{P}), \\
\text { rods }\end{array}$ & $\begin{array}{l}(\mathrm{N}), \\
\text { rods }\end{array}$ \\
\hline Indole & $\mathrm{N}$ & $\mathrm{N}$ & $\mathrm{N}$ & $\mathrm{N}$ & $\mathrm{N}$ & $\mathrm{N}$ & $\mathrm{N}$ & $\mathrm{N}$ & $\mathrm{N}$ & $\mathrm{N}$ & $\mathrm{N}$ & $\mathrm{N}$ & $\mathrm{N}$ & $\mathrm{N}$ & $\mathrm{N}$ & $\mathrm{N}$ & $\mathrm{N}$ & $\mathrm{N}$ & $\mathrm{N}$ & $\mathrm{N}$ & $\mathrm{N}$ \\
\hline MR & $\mathrm{P}$ & $\mathrm{P}$ & $\mathrm{N}$ & $\mathrm{P}$ & $\mathrm{N}$ & $\mathrm{P}$ & $\mathrm{N}$ & $\mathrm{P}$ & $\mathrm{N}$ & $\mathrm{P}$ & $\mathrm{N}$ & $\mathrm{P}$ & $\mathrm{N}$ & $\mathrm{P}$ & $\mathrm{N}$ & $\mathrm{P}$ & $\mathrm{N}$ & $\mathrm{P}$ & $\mathrm{N}$ & $\mathrm{P}$ & $\mathrm{N}$ \\
\hline VP & $\mathrm{P}$ & $\mathrm{P}$ & $\mathrm{P}$ & $\mathrm{P}$ & $\mathrm{P}$ & $\mathrm{P}$ & $\mathrm{P}$ & $\mathrm{P}$ & $\mathrm{P}$ & $\mathrm{P}$ & $\mathrm{P}$ & $\mathrm{P}$ & $\mathrm{P}$ & $\mathrm{P}$ & $\mathrm{P}$ & $\mathrm{P}$ & $\mathrm{P}$ & $\mathrm{P}$ & $\mathrm{P}$ & $\mathrm{P}$ & $\mathrm{P}$ \\
\hline Citrate & $\mathrm{P}$ & $\mathrm{P}$ & $\mathrm{N}$ & $\mathrm{P}$ & $\mathrm{N}$ & $\mathrm{P}$ & $\mathrm{N}$ & $\mathrm{P}$ & $\mathrm{N}$ & $\mathrm{P}$ & $\mathrm{N}$ & $\mathrm{P}$ & $\mathrm{N}$ & $\mathrm{P}$ & $\mathrm{N}$ & $\mathrm{P}$ & $\mathrm{N}$ & $\mathrm{P}$ & $\mathrm{N}$ & $\mathrm{P}$ & $\mathrm{N}$ \\
\hline TSI & $\mathrm{P}$ & $\mathrm{P}$ & $\mathrm{N}$ & $\mathrm{P}$ & $\mathrm{N}$ & $\mathrm{P}$ & $\mathrm{N}$ & $\mathrm{P}$ & $\mathrm{N}$ & $\mathrm{P}$ & $\mathrm{N}$ & $\mathrm{P}$ & $\mathrm{N}$ & $\mathrm{P}$ & $\mathrm{N}$ & $\mathrm{P}$ & $\mathrm{N}$ & $\mathrm{P}$ & $\mathrm{N}$ & $\mathrm{P}$ & $\mathrm{N}$ \\
\hline Nitrate & $\mathrm{N}$ & $\mathrm{N}$ & $\mathrm{N}$ & $\mathrm{N}$ & $\mathrm{N}$ & $\mathrm{N}$ & $\mathrm{N}$ & $\mathrm{N}$ & $\mathrm{N}$ & $\mathrm{N}$ & $\mathrm{N}$ & $\mathrm{N}$ & $\mathrm{N}$ & $\mathrm{N}$ & $\mathrm{N}$ & $\mathrm{N}$ & $\mathrm{N}$ & $\mathrm{N}$ & $\mathrm{N}$ & $\mathrm{N}$ & $\mathrm{N}$ \\
\hline Catalase test & $\mathrm{P}$ & $\mathrm{P}$ & $\mathrm{P}$ & $\mathrm{P}$ & $\mathrm{P}$ & $\mathrm{P}$ & $\mathrm{P}$ & $\mathrm{P}$ & $\mathrm{P}$ & $\mathrm{P}$ & $\mathrm{P}$ & $\mathrm{P}$ & $\mathrm{P}$ & $\mathrm{P}$ & $\mathrm{P}$ & $\mathrm{P}$ & $\mathrm{P}$ & $\mathrm{P}$ & $\mathrm{P}$ & $\mathrm{P}$ & $\mathrm{P}$ \\
\hline Motility & $\mathrm{P}$ & $\mathrm{P}$ & $\mathrm{N}$ & $\mathrm{P}$ & $\mathrm{N}$ & $\mathrm{P}$ & $\mathrm{N}$ & $\mathrm{P}$ & $\mathrm{N}$ & $\mathrm{P}$ & $\mathrm{N}$ & $\mathrm{P}$ & $\mathrm{N}$ & $\mathrm{P}$ & $\mathrm{N}$ & $\mathrm{P}$ & $\mathrm{N}$ & $\mathrm{P}$ & $\mathrm{N}$ & $\mathrm{P}$ & $\mathrm{N}$ \\
\hline Mannitol fermentation & $\mathrm{N}$ & $\mathrm{N}$ & $\mathrm{N}$ & $\mathrm{N}$ & $\mathrm{N}$ & $\mathrm{N}$ & $\mathrm{N}$ & $\mathrm{N}$ & $\mathrm{N}$ & $\mathrm{N}$ & $\mathrm{N}$ & $\mathrm{N}$ & $\mathrm{N}$ & $\mathrm{N}$ & $\mathrm{N}$ & $\mathrm{N}$ & $\mathrm{N}$ & $\mathrm{N}$ & $\mathrm{N}$ & $\mathrm{N}$ & $\mathrm{N}$ \\
\hline Xylose fermentation & $\mathrm{P}$ & $\mathrm{P}$ & $\mathrm{N}$ & $\mathrm{P}$ & $\mathrm{N}$ & $\mathrm{P}$ & $\mathrm{N}$ & $\mathrm{P}$ & $\mathrm{N}$ & $\mathrm{P}$ & $\mathrm{N}$ & $\mathrm{P}$ & $\mathrm{N}$ & $\mathrm{P}$ & $\mathrm{N}$ & $\mathrm{P}$ & $\mathrm{N}$ & $\mathrm{P}$ & $\mathrm{N}$ & $\mathrm{P}$ & $\mathrm{N}$ \\
\hline Glucose fermentation & $\mathrm{P}$ & $\mathrm{P}$ & $\mathrm{N}$ & $\mathrm{P}$ & $\mathrm{N}$ & $\mathrm{P}$ & $\mathrm{N}$ & $\mathrm{P}$ & $\mathrm{N}$ & $\mathrm{P}$ & $\mathrm{N}$ & $\mathrm{P}$ & $\mathrm{N}$ & $\mathrm{P}$ & $\mathrm{N}$ & $\mathrm{P}$ & $\mathrm{N}$ & $\mathrm{P}$ & $\mathrm{N}$ & $\mathrm{P}$ & $\mathrm{N}$ \\
\hline Fructose fermentation & $\mathrm{P}$ & $\mathrm{P}$ & $\mathrm{N}$ & $\mathrm{P}$ & $\mathrm{N}$ & $\mathrm{P}$ & $\mathrm{N}$ & $\mathrm{P}$ & $\mathrm{N}$ & $\mathrm{P}$ & $\mathrm{N}$ & $\mathrm{P}$ & $\mathrm{N}$ & $\mathrm{P}$ & $\mathrm{N}$ & $\mathrm{P}$ & $\mathrm{N}$ & $\mathrm{P}$ & $\mathrm{N}$ & $\mathrm{P}$ & $\mathrm{N}$ \\
\hline Galactose fermentation & $\mathrm{P}$ & $\mathrm{P}$ & $\mathrm{N}$ & $\mathrm{P}$ & $\mathrm{N}$ & $\mathrm{P}$ & $\mathrm{N}$ & $\mathrm{P}$ & $\mathrm{N}$ & $\mathrm{P}$ & $\mathrm{N}$ & $\mathrm{P}$ & $\mathrm{N}$ & $\mathrm{P}$ & $\mathrm{N}$ & $\mathrm{P}$ & $\mathrm{N}$ & $\mathrm{P}$ & $\mathrm{N}$ & $\mathrm{P}$ & $\mathrm{N}$ \\
\hline Maltose fermentation & $\mathrm{N}$ & $\mathrm{N}$ & $\mathrm{N}$ & $\mathrm{N}$ & $\mathrm{N}$ & $\mathrm{N}$ & $\mathrm{N}$ & $\mathrm{N}$ & $\mathrm{N}$ & $\mathrm{N}$ & $\mathrm{N}$ & $\mathrm{N}$ & $\mathrm{N}$ & $\mathrm{N}$ & $\mathrm{N}$ & $\mathrm{N}$ & $\mathrm{N}$ & $\mathrm{N}$ & $\mathrm{N}$ & $\mathrm{N}$ & $\mathrm{N}$ \\
\hline Lactose fermentation & $\mathrm{P}$ & $\mathrm{P}$ & $\mathrm{N}$ & $\mathrm{P}$ & $\mathrm{N}$ & $\mathrm{P}$ & $\mathrm{N}$ & $\mathrm{P}$ & $\mathrm{N}$ & $\mathrm{P}$ & $\mathrm{N}$ & $\mathrm{P}$ & $\mathrm{N}$ & $\mathrm{P}$ & $\mathrm{N}$ & $\mathrm{P}$ & $\mathrm{N}$ & $\mathrm{P}$ & $\mathrm{N}$ & $\mathrm{P}$ & $\mathrm{N}$ \\
\hline
\end{tabular}

Abbreviation: P: Test Positive and, N: Test Negative, A1 to A20: 20 individual samples of Labeo catla. 
Table.3 Cultural and biochemical characteristics of Listeria species isolated from Dasyatis pastinaca

\begin{tabular}{|c|c|c|c|c|c|c|c|c|c|c|c|c|c|c|c|c|c|c|c|c|c|}
\hline $\begin{array}{c}\text { Dasyatis pastinaca } \\
\text { Isolates }\end{array}$ & $\begin{array}{c}\text { ATCC } \\
7644\end{array}$ & B1 & B2 & B3 & B4 & B5 & B6 & B7 & B8 & B9 & B10 & B11 & B12 & B13 & B14 & B15 & B16 & B17 & B18 & B19 & B20 \\
\hline Gram's staining & $\begin{array}{l}(\mathrm{P}) \\
\text { rods }\end{array}$ & $\begin{array}{l}(\mathrm{N}), \\
\operatorname{rod}\end{array}$ & $\begin{array}{l}(\mathrm{P}), \\
\text { rods }\end{array}$ & $\begin{array}{l}(\mathrm{P}) \\
\text { cocci }\end{array}$ & $\begin{array}{l}(\mathrm{P}), \\
\text { rods }\end{array}$ & $\begin{array}{l}(\mathrm{N}), \\
\text { rods }\end{array}$ & $\begin{array}{l}(\mathrm{P}), \\
\text { rods }\end{array}$ & $\begin{array}{l}(\mathrm{N}), \\
\text { rods }\end{array}$ & $\begin{array}{l}(\mathrm{P}), \\
\text { rods }\end{array}$ & $\begin{array}{l}(\mathrm{P}), \\
\text { rods }\end{array}$ & $\begin{array}{l}(\mathrm{P}), \\
\text { rods }\end{array}$ & $\begin{array}{l}(\mathrm{N}) \\
\operatorname{rod}\end{array}$ & $\begin{array}{l}(\mathrm{P}), \\
\text { rods }\end{array}$ & $\begin{array}{l}(\mathrm{P}) \\
\text { cocci }\end{array}$ & $\begin{array}{l}(\mathrm{P}), \\
\text { rods }\end{array}$ & $\begin{array}{l}(\mathrm{N}), \\
\text { rods }\end{array}$ & $\begin{array}{l}(\mathrm{P}), \\
\text { rods }\end{array}$ & $\begin{array}{l}(\mathrm{N}), \\
\text { rods }\end{array}$ & $\begin{array}{l}(\mathrm{P}), \\
\text { rods }\end{array}$ & $\begin{array}{l}(\mathrm{P}), \\
\text { rods }\end{array}$ & $\begin{array}{l}(\mathrm{P}), \\
\text { rods }\end{array}$ \\
\hline Indole & $\mathrm{N}$ & $\mathrm{N}$ & $\mathrm{N}$ & $\mathrm{N}$ & $\mathrm{N}$ & $\mathrm{N}$ & $\mathrm{N}$ & $\mathrm{N}$ & $\mathrm{N}$ & $\mathrm{N}$ & $\mathrm{N}$ & $\mathrm{N}$ & $\mathrm{N}$ & $\mathrm{N}$ & $\mathrm{N}$ & $\mathrm{N}$ & $\mathrm{N}$ & $\mathrm{N}$ & $\mathrm{N}$ & $\mathrm{N}$ & $\mathrm{N}$ \\
\hline MR & $\mathrm{P}$ & $\mathrm{N}$ & $\mathrm{P}$ & $\mathrm{N}$ & $\mathrm{P}$ & $\mathrm{N}$ & $\mathrm{P}$ & $\mathrm{N}$ & $\mathrm{P}$ & $\mathrm{N}$ & $\mathrm{P}$ & $\mathrm{N}$ & $\mathrm{P}$ & $\mathrm{N}$ & $\mathrm{P}$ & $\mathrm{N}$ & $\mathrm{P}$ & $\mathrm{N}$ & $\mathrm{P}$ & $\mathrm{N}$ & $\mathrm{P}$ \\
\hline VP & $\mathrm{P}$ & $\mathrm{P}$ & $\mathrm{P}$ & $\mathrm{P}$ & $\mathrm{P}$ & $\mathrm{P}$ & $\mathrm{P}$ & $\mathrm{P}$ & $\mathrm{P}$ & $\mathrm{P}$ & $\mathrm{P}$ & $\mathrm{P}$ & $\mathrm{P}$ & $\mathrm{P}$ & $\mathrm{P}$ & $\mathrm{P}$ & $\mathrm{P}$ & $\mathrm{P}$ & $\mathrm{P}$ & $\mathrm{P}$ & $\mathrm{P}$ \\
\hline Citrate & $\mathrm{P}$ & $\mathrm{N}$ & $\mathrm{P}$ & $\mathrm{N}$ & $\mathrm{P}$ & $\mathrm{N}$ & $\mathrm{P}$ & $\mathrm{N}$ & $\mathrm{P}$ & $\mathrm{N}$ & $\mathrm{P}$ & $\mathrm{N}$ & $\mathrm{P}$ & $\mathrm{N}$ & $\mathrm{P}$ & $\mathrm{N}$ & $\mathrm{P}$ & $\mathrm{N}$ & $\mathrm{P}$ & $\mathrm{N}$ & $\mathrm{P}$ \\
\hline TSI & $\mathrm{P}$ & $\mathrm{N}$ & $\mathrm{P}$ & $\mathrm{N}$ & $\mathrm{P}$ & $\mathrm{N}$ & $\mathrm{P}$ & $\mathrm{N}$ & $\mathrm{P}$ & $\mathrm{N}$ & $\mathrm{P}$ & $\mathrm{N}$ & $\mathrm{P}$ & $\mathrm{N}$ & $\mathrm{P}$ & $\mathrm{N}$ & $\mathrm{P}$ & $\mathrm{N}$ & $\mathrm{P}$ & $\mathrm{N}$ & $\mathrm{P}$ \\
\hline Nitrate & $\mathrm{N}$ & $\mathrm{N}$ & $\mathrm{N}$ & $\mathrm{N}$ & $\mathrm{N}$ & $\mathrm{N}$ & $\mathrm{N}$ & $\mathrm{N}$ & $\mathrm{N}$ & $\mathrm{N}$ & $\mathrm{N}$ & $\mathrm{N}$ & $\mathrm{N}$ & $\mathrm{N}$ & $\mathrm{N}$ & $\mathrm{N}$ & $\mathrm{N}$ & $\mathrm{N}$ & $\mathrm{N}$ & $\mathrm{N}$ & $\mathrm{N}$ \\
\hline Catalase & $\mathrm{P}$ & $\mathrm{P}$ & $\mathrm{P}$ & $\mathrm{P}$ & $\mathrm{P}$ & $\mathrm{P}$ & $\mathrm{P}$ & $\mathrm{P}$ & $\mathrm{P}$ & $\mathrm{P}$ & $\mathrm{P}$ & $\mathrm{P}$ & $\mathrm{P}$ & $\mathrm{P}$ & $\mathrm{P}$ & $\mathrm{P}$ & $\mathrm{P}$ & $\mathrm{P}$ & $\mathrm{P}$ & $\mathrm{P}$ & $\mathrm{P}$ \\
\hline Motility & $\mathrm{P}$ & $\mathrm{N}$ & $\mathrm{P}$ & $\mathrm{N}$ & $\mathrm{P}$ & $\mathrm{N}$ & $\mathrm{P}$ & $\mathrm{N}$ & $\mathrm{P}$ & $\mathrm{N}$ & $\mathrm{P}$ & $\mathrm{N}$ & $\mathrm{P}$ & $\mathrm{N}$ & $\mathrm{P}$ & $\mathrm{N}$ & $\mathrm{P}$ & $\mathrm{N}$ & $\mathrm{P}$ & $\mathrm{N}$ & $\mathrm{P}$ \\
\hline Mannitol fermentation & $\mathrm{N}$ & $\mathrm{N}$ & $\mathrm{N}$ & $\mathrm{N}$ & $\mathrm{N}$ & $\mathrm{N}$ & $\mathrm{N}$ & $\mathrm{N}$ & $\mathrm{N}$ & $\mathrm{N}$ & $\mathrm{N}$ & $\mathrm{N}$ & $\mathrm{N}$ & $\mathrm{N}$ & $\mathrm{N}$ & $\mathrm{N}$ & $\mathrm{N}$ & $\mathrm{N}$ & $\mathrm{N}$ & $\mathrm{N}$ & $\mathrm{N}$ \\
\hline Xylose fermentation & $\mathrm{P}$ & $\mathrm{N}$ & $\mathrm{P}$ & $\mathrm{N}$ & $\mathrm{P}$ & $\mathrm{N}$ & $\mathrm{P}$ & $\mathrm{N}$ & $\mathrm{P}$ & $\mathrm{N}$ & $\mathrm{P}$ & $\mathrm{N}$ & $\mathrm{P}$ & $\mathrm{N}$ & $\mathrm{P}$ & $\mathrm{N}$ & $\mathrm{P}$ & $\mathrm{N}$ & $\mathrm{P}$ & $\mathrm{N}$ & $\mathrm{P}$ \\
\hline Glucose fermentation & $\mathrm{P}$ & $\mathrm{N}$ & $\mathrm{P}$ & $\mathrm{N}$ & $\mathrm{P}$ & $\mathrm{N}$ & $\mathrm{P}$ & $\mathrm{N}$ & $\mathrm{P}$ & $\mathrm{N}$ & $\mathrm{P}$ & $\mathrm{N}$ & $\mathrm{P}$ & $\mathrm{N}$ & $\mathrm{P}$ & $\mathrm{N}$ & $\mathrm{P}$ & $\mathrm{N}$ & $\mathrm{P}$ & $\mathrm{N}$ & $\mathrm{P}$ \\
\hline Fructose fermentation & $\mathrm{P}$ & $\mathrm{N}$ & $\mathrm{P}$ & $\mathrm{N}$ & $\mathrm{P}$ & $\mathrm{N}$ & $\mathrm{P}$ & $\mathrm{N}$ & $\mathrm{P}$ & $\mathrm{N}$ & $\mathrm{P}$ & $\mathrm{N}$ & $\mathrm{P}$ & $\mathrm{N}$ & $\mathrm{P}$ & $\mathrm{N}$ & $\mathrm{P}$ & $\mathrm{N}$ & $\mathrm{P}$ & $\mathrm{N}$ & $\mathrm{P}$ \\
\hline Galactose fermentation & $\mathrm{P}$ & $\mathrm{N}$ & $\mathrm{P}$ & $\mathrm{N}$ & $\mathrm{P}$ & $\mathrm{N}$ & $\mathrm{P}$ & $\mathrm{N}$ & $\mathrm{P}$ & $\mathrm{N}$ & $\mathrm{P}$ & $\mathrm{N}$ & $\mathrm{P}$ & $\mathrm{N}$ & $\mathrm{P}$ & $\mathrm{N}$ & $\mathrm{P}$ & $\mathrm{N}$ & $\mathrm{P}$ & $\mathrm{N}$ & $\mathrm{P}$ \\
\hline Maltose fermentation & $\mathrm{N}$ & $\mathrm{N}$ & $\mathrm{N}$ & $\mathrm{N}$ & $\mathrm{N}$ & $\mathrm{N}$ & $\mathrm{N}$ & $\mathrm{N}$ & $\mathrm{N}$ & $\mathrm{N}$ & $\mathrm{N}$ & $\mathrm{N}$ & $\mathrm{N}$ & $\mathrm{N}$ & $\mathrm{N}$ & $\mathrm{N}$ & $\mathrm{N}$ & $\mathrm{N}$ & $\mathrm{N}$ & $\mathrm{N}$ & $\mathrm{N}$ \\
\hline Lactose fermentation & $\mathrm{P}$ & $\mathrm{N}$ & $\mathrm{P}$ & $\mathrm{N}$ & $\mathrm{P}$ & $\mathrm{N}$ & $\mathrm{P}$ & $\mathrm{N}$ & $\mathrm{P}$ & $\mathrm{N}$ & $\mathrm{P}$ & $\mathrm{N}$ & $\mathrm{P}$ & $\mathrm{N}$ & $\mathrm{P}$ & $\mathrm{N}$ & $\mathrm{P}$ & $\mathrm{N}$ & $\mathrm{P}$ & $\mathrm{N}$ & $\mathrm{P}$ \\
\hline
\end{tabular}

Abbreviation: P: Test Positive and, N: Test Negative, B1 to B20: 20 individual samples of Dasyatis pastinaca. 
Our results demonstrate that the two different type of fishes obtained from Chennai west retail shops contains $L$. monocytogenes, which is observed as an important human pathogen, in their intestines. Intestinal guts of fish generally infect the fish meat and this causes the contamination of foodborne pathogens such as L. monocytogenes during the factoring process. The occurrence of L. monocytogenes in freshwater fish was $6.6 \%$. This rate is lower than that in a maximum of the published studies on fresh fish. In a study, 20 samples of Labeo catla and 20 samples of Dasyatis pastinaca were analyzed for the presence of Listeria spp. and $L$. monocytogenes.

The frequency of Listeria and $L$. monocytogenes in Labeo catla and Dasyatis pastinaca was $50 \%$ and $50 \%$, respectively. Miettinen et al., (2001) studied the external contamination and the occurrence of $L$. monocytogenes in fish dispensation factories. Listeria spp. was determined at a level of $45 \%$ and $L$. monocytogenes with at a level of $12 \%$. In a different experiment, 56 fresh fish samples were analyzed for the occurrence of Listeria spp. and 15 of the samples were identified to be contaminated with $L$. monocytogenes and L. innocua were isolated from 3 and 12 samples, correspondingly (Vaz-Velho et al., 2001). Fuchs and Surendran (1989), were unable to isolate $L$. monocytogenes in fresh fish from India, while $33 \%$ of the samples harbored Listeria spp. Conversely, Adesiyun (1993) documented a $2 \%$ occurrence of L. monocytogenes in fish and shellfish in India.

Comparatively, the outcomes of this experiment were higher than those of other, related experiments. All fish used in this experiment were acquired from the same area of the west Chennai retail shops and the sewage system of the area had been quitting into this region for several months. Hence, the isolation rate was anticipated to be high. Still, biological cleaning might not have been executed before discarding into the water bodies and this might have caused the isolation rate to be higher than that in other results.

The procedures used for the isolation of $L$. monocytogenes from foods materials are different. Many techniques involved in the enrichment and selective plating methods. The media used for enrichment and selective plating have also different methods (Karunasagar et al., 2000). The procedure accepted by the standard ISO: 11290 (1996) method was used for the isolation of $L$. myonocytogenes in this study and followed by cultural, biochemical and molecular characterization and confirmation of isolates.

Listeria spp. was isolated from $50 \%(20 / 40)$ of the sample of fish with the highest prevalence from fish samples. Intake of these fish foods, either raw or undercooked, may contribute to food-borne illness due to $L$. monocytogenes. L. monocytogenes in raw seafood may pose a health risk in pantries if contaminating ready to eat food. However, diseases could also get transmitted through the consumption of contaminated foods of animal origin and the extent of their occurrence has shown an increasing trend over the years.

In summary, our outcomes showed that the fish from Chennai west hypermarkets and street hawkers contain L. monocytogenes in their intestines and these fish may cause Listeriosis outbreaks as reported previously (Miettinen et al., 1999 and Ericsson et al., 1997). Humans consuming the contaminated fish and their foods are at risk of disease. The genotyping results indicated that the strains isolated have diverse genetic profiles. This may be the consequence of different sources of bacteria. More discriminative typing 
methods could be used to disclose the relationship between human and fish isolates.

The high prevalence of $L$. monocytogenes in fish foods sold by hypermarkets and street hawkers pose a high risk to the consumer. Since fish foods are consumed directly after purchase with no further treatment applied to reduce Listeria in fish foods. Therefore, the materials have to maintain proper temperature before the sale and production of safe foods for the consumers. However, if one aims to detect Listeria spp. but not just $L$. monocytogenes, the combination use of two to three plating media are required to achieve higher proficiency of Listeria spp. detection.

\section{References}

Adesiyun, A.A. 1993. Prevalence of Listeria spp., Campylobacter spp. and toxigenic Escherichia coli on meat and seafoods in Trinidad. Food Microbiol 10(5): 395403.

Alsheikh, A.D.I., Mohammed, G.E. and Abdalla, M.A. 2013. Isolation and identification of Listeria monocytogenes from retail broiler chicken Ready to Eat Meat Products in Sudan. Int. J. Anim. Veter. Adv 5(1): 9-14.

Borucki, M.K., Peppin, J.D., White, D., Loge, F. and Call, D.R. 2003. Variation in biofilm formation among strains of Listeria monocytogenes. Appl. Environ. Microbiol 69(12): 7336-7342.

Byun, S.K., Jung, S.C. and Yoo, H.S. 2001. Random amplification of polymorphic DNA typing of Listeria monocytogenes isolated from meat. Int. J. Food. Microbiol 69(3): 227-235.

Edgar, R.C. 2004. MUSCLE: multiple sequence alignment with high accuracy and high throughput. Nucleic. Acids. Res 32(5): 1792-1797.

Edwards, U., Rogall, T., Blocker, H., Emde, M. and Bottger, E.C. 1989. Isolation and direct complete nucleotide determination of entire genes: characterization of a gene coding for16S ribosomal RNA. Nucleic Acids Res 17(19): 7843-7853.

Ericsson, H., Eklow, A., Danielsson-Tham, M.L., Loncarevic, S., Mentzing, L.O., Persson, I., Unnerstad, H. and Tham, W. 1997. An outbreak of listeriosis suspected to have been caused by rainbow trout. J. Clin. Microbiol 35(11): 2904-2907.

Farber, J.M. and Peterkin, P.I. 1991. Listeria monocytogenes, a food borne pathogen. Microbiol. Rev 55(3): 476-511.

Fuchs, R.S. and Surendran, P.K. 1989. Incidence of Listeria in tropical fish and fishery products. Lett. Appl. Microbiol 9(2): 49-51.

Gillian, A.F. and David, O. 2001. Effects of acid adaptation on the survival of Listeria monocytogenes on modified atmosphere packaged vegetables. International Journal of Food Science \& Technology 36(5): 477-487.

Jrgensen, L.V. and Huss, H.H. 1998. Prevalence and growth of Listeria monocytogenes in naturally contaminated seafood. Int. J. Food Microbiol 42(1-2): 127-131.

Karunasagar, I. and Karunasagar, I. 2000. Listeria in tropical fish and fishery products. Int. J. Food Microbiol 62(3): 177-181.

Miettinen, H., Aarnisalo, K., Salo, S. and Sjoberg, A.M. 2001. Evaluation of surface contamination and the presence of Listeria monocytogenes in fish processing factories. J. Food Protect 64(5): 635-639.

Miettinen, M.K., Siitonen, A., Heiskanen, P., Haajanen. H., Bjorkroth, K.J. and Korkeala, H.J. 1999. Molecular epidemiology of an outbreak of febrile gastroenteritis caused by Listeria monocytogenes in cold-smoked rainbow 
trout. J. Clin. Microbiol 37(7): 23582360.

Schuchat, A., Swaminathan, B. and Broome, C.V. 1991. Epidemiology of human listeriosis. Clin. Microbiol. Rev 4(3): $169-183$.

Seeliger, H.P.R. and Hohne, K. 1979. Serotyping of Listeria monocytogenes and related species. Methods in Microbiology 13: 31-49.

Talavera, G. and Castresana, J. 2007. Improvement of phylogenies after removing divergent and ambiguously aligned blocks from protein sequence alignments. Syst. Biol 56(4): 564-577.

Vaz-Velho, M., Duarte, G., McLauchlin, J. and Gibbs, P. 2001. Characterization of Listeria monocytogenes isolated from production lines of fresh and coldsmoked fish. J. Appl. Microbiol 91(3): $556-562$.

Walker, S.F., Archer, P. and Banks, J.G. 1990. Growth of Listeria monocytogenes at refrigeration temperatures. J. Appl. Bacteriol 68(2): 20-23.

\section{How to cite this article:}

Madharsha, N., Veeramanikandan Veeramani, Jaianand Kannaiyan and Balaji Paulraj. 2018. Isolation and Identification of Listeria monocytogenes from Fish Intestines and Phylogenetic Analysis. Int.J.Curr.Microbiol.App.Sci. 7(11): 1177-1187. doi: https://doi.org/10.20546/ijcmas.2018.711.137 